https://doi.org/10.46344/JBINO.2020.v09i04.27

\title{
COVID-19: ORIGINAL SIMPLE AND CHEAP EXTRAPULMONARY OXYGENATION AS AN ALTERNATIVE TO ECMO-Review Article
}

-Local hypothermic ischemia of limbs with gastric hyperoxygenation is the last way to prevent tragedy COVID-19 before ECMO.

\section{1,2A.L.Urakov}

'Department of Modeling and Synthesis of Technological Structures Udmurt Federal Research Center of the Ural branch Russian Academy of Sciences; 2Department of General and Clinical Pharmacology of the Izhevsk State Medical Academy of the Ministry of health Russian Federation, Izhevsk, Russia - 426034

E-mail: urakoval@live.ru

(Received on Date: 20 June 2020 Date of Acceptance: 30th June 2020 Date of Publish: 01 st July 2020)

\section{ABSTRACT}

It is shown that ECMO is used today to protect the brain from hypoxic damage with low efficiency of pulmonary ventilation in COVID-19. But traditional ECMO is a very complex, expensive and dangerous procedure. Therefore, not all patients and not always manage to apply ECMO in a timely manner. Moreover, even if ECMO is used, it is always advisable to reduce the duration of this procedure by a few days or hours. In this regard, a modernized method of extrapulmonary oxygenation in combination with General artificial hypothermia and/or local craniocerebral hypothermia is proposed as an alternative to ECMO. This backup method of saving life involves injecting a solution of hydrogen peroxide into the patient's stomach, carbonated with oxygen under excessive pressure, and simultaneously applying tourniquets to the extremities with their local cooling to $+18{ }^{\circ} \mathrm{C}$. Additionally, general artificial hypothermia and/or craniocerebral hypothermia can be used. At the same time, the introduction of hydrogen peroxide and oxygen gas into the stomach provides extrapulmonary oxygenation, the creation of acute cold limb ischemia provides artificial hypercentration of blood circulation, and general and/or craniocerebral hypothermia reduces the brain's need for oxygen.

Key words: ARDS, SARS-CoV-2, COVID-19, pneumonia, refractory pulmonary failure, oxygenation.

No:of References: 24 


\section{Introduction}

It has been shown that death in the tragic end of a disease caused by a new coronavirus (COVID-19) occurs due to a lack of oxygen for the brain ${ }^{1,2}$. It is established that hypoxia develops due to acute respiratory distress syndrome (ARDS), which, in turn, develops as a result of two-way pneumonia ${ }^{3}$. It is generally recognized that for timely diagnosis of COVID-19, it is most important to perform a CT scan of the lungs, which can detect non-specific pneumonia and double-sided "frosted glass opacity" in the lungs 4,5 . The fact is that the detection of "frosted glass opacity" in the lungs during x-ray examination coincides with the most severe degree of the disease ${ }^{6}$. And because in such cases, patients are not able to independently remove thick pus and thick mucus from the airway lumen?. That is why when x-ray detection of the "frosted glass opacity" syndrome in a patient, it is necessary to be prepared for the need to eliminate brain hypoxia in them. Currently, extracorporeal membrane oxygenation (ECMO) is used for this purpose.

However, ECMO technology is not available to everyone in need, as this treatment technology is very complex and expensive. Regardless of this, the technology of ECMO is very dangerous for patients ${ }^{8}$. The fact is that the death rate from ECMO in respiratory failure can often exceed $50 \%$. At the same time, ECMO costs between $\$ 5,000$ and $\$$ 10,000 per day ${ }^{10}$. But, there is no generally accepted alternative for ECMO yet. Therefore, the death rate from ARDS when COVID-19 remains high even in those countries where ECMO is most widely used. In this regard, the search and development of new ways to save lives in ARDS remains a very urgent task. The aim of the study is to develop an alternative to ECMO in the form of extrapulmonary oxygenation.

\section{Methods}

Scientific and patent literature was analyzed using Google Patent, Google Scholar, Scopus, and PubMed databases. Keywords of the search strategy were as follows: ARDS, SARS-COV-2, COVID-19, pneumonia, refractory pulmonary failure, oxygenation, pulmonary insufficiency, drowning, apnea, brain, hypoxia, ischemia, resistance to hypoxia, adaptation to hypoxia, oxygen, hydrogen peroxide, aerobic exchange, pulmonary oxygenation, extrapulmonary oxygenation, blood volume, hypothermia, blood loss, adaptation to blood loss, blood substitutes, lymph substitutes, thick pus solvents, thick mucus solvents, aerosols. Keywords were limited to the means and methods of emergency care in critical situations in pulmonology and resuscitation. The results were analyzed, prioritized, and summarized.

\section{Results}

Previously, we suggested that it is possible to save the life of patients with ARDS when COVID-19 by using a special aerosol with Hydrogen peroxide that quickly dissolves thick pus and mucus in the airways, facilitates their removal and restores the patency of the bronchi and bronchioles for inhaled air 1 . To do this, it was proposed to use a solution of $1.2 \%$ Sodium hydrocarbonate, $\quad 0.3-0.5 \%$ Hydrogen peroxide and $0.5 \%$ Lidocaine hydrochloride at a temperature of +41 $+55{ }^{\circ} \mathrm{C}$ (RU Application N 2020100533. 
09.01.2020). It was proposed to use of aerosol by dispersed spraying of liquids using ultrasonic, compression and jet inhalers and nebulizers.

The advantage of such an aerosol is that it releases a gas - molecular oxygen. It is also important that the main ingredient in the formulation of this aerosol is hydrogen peroxide, which has long time been used in medicine and is an antiseptic. It is mainly used as an aqueous solution of $3 \%$ hydrogen peroxide in treatment of purulent wounds and/or to sterilize irrigated surfaces ${ }^{11}$.

However, the legal and safe treatment of patients by inhalation of an invented aerosol will be possible only after completing the entire volume of necessary clinical trials. It is obvious that clinical research will require a lot of time and money. In this situation, everyone has the right to ask the question, how can we help dying with COVID-19 patients today without violating the law, if it is known that patients with ARDS die from lack of oxygen for the brain? After all, it follows that in order to urgently save the life of a patient with ARDS when COVID-19, it is necessary to urgently increase the delivery of oxygen to the brain. Is there no oxygen reserves inside the patient if pulmonary oxygenation is temporarily impossible? In this case, increasing the delivery of oxygen to the brain, which will be achieved by "necessary" redistribution of oxygen inside the body of the dying patient, will not be a violation of the law. Especially if a method that has long been used in medicine is used for this purpose.

In addition, regardless of the answer to first question, everyone can ask a second question about whether it is possible to increase the brain's resistance to hypoxia and/or reduce its oxygen demand without medication? A positive answer to this question is very important, because in this case we will also not need to spend time on clinical trials of drugs, and we will be able to help patients with coronavirus pneumonia, bronchial obstruction and ARDS today.

It has been shown that in situations where traditional ECMO using gas oxygen can't be applied, and hypoxia really threatens the life of a patient with pneumonia and obstructive bronchitis caused by COVID-19, blood oxygenation is possible with a solution of hydrogen peroxide ${ }^{12,13}$. The fact is that a solution of hydrogen peroxide can compete with oxygen gas during resuscitation in critical situations ${ }^{13,14}$. In particular, it was shown that this drug can be introduced into the intestines with an enema or with an intestinal probe. Extrapulmonary oxygenation was studied in dogs, rabbits, and finally in the case of Fallot tetralogy in a patient using intestinal perfusion of hydrogen peroxide solution ${ }^{15}$. It was shown that a single injection of $0.2-0.4 \%$ $\mathrm{H}_{2} \mathrm{O}_{2}$ solution into the intestine with an enema at a dose of $10 \mathrm{ml} / \mathrm{kg}$ of body weight is completely safe and allows oxygenation of blood in the portal vein and in the inferior vena cava without the formation of a gas embolism.

In addition, the "Method for extrapulmonary blood oxygenation" (RU Patent 2505323. 27.01.2014) was invented, in which for extrapulmonary blood oxygenation, it was proposed to inject oxygen gas into the 12-duodenum using a probe with a volumetric speed in the range of 10-150 ml per minute. Other authors in experiments on adult rabbits have shown that peritoneal oxygenation 
effectively reduces hypoxia in experimental lung failure caused by drowning rabbits in water ${ }^{16}$.

In addition, in experiments with aquarium fish and in studies on adult healthy volunteers, it was shown that adaptation to acute hypoxia can be increased by introducing a solution of hydrogen peroxide into the stomach. Moreover, to increase oxygenation, it was proposed to saturate the solution of hydrogen peroxide with oxygen gas under an excessive dilution of 0.2 ATM. This method of oxygenating the blood has been called "intragastric blood oxygenation"13,17.

In parallel with the development of methods for extrapulmonary blood oxygenation, research was conducted to increase the resistance of the cerebral cortex to hypoxia in hemorrhagic shock in adults and in intrauterin fetal hypoxia. The results of these studies led to the belief that the resistance of cortical cells to acute hypoxia increases hypothermia ${ }^{18}$ and the centralization of blood circulation ${ }^{19}$.

At the same time, more than 30 years ago, it was proved that local cooling of the limbs and heart increases their resistance to acute ischemia ${ }^{20,21}$. The following is very important: should pay attention to the simplicity and cheapness of the treatment method, namely, local cooling. Therefore, it was decided to use these scientific data to modernize the known methods of extrapulmonary oxygenation. At the same time, the main task of the planned modernization was to maintain simplicity and cheapness, since traditional ECMO is not only dangerous for the patient, but also very expensive and complex ${ }^{22}$.
First, to preserve the simplicity of the method of extrapulmonary oxygenation, there is obviously no alternative to using a solution of hydrogen peroxide saturated with gas oxygen $\left(\mathrm{O}_{2}\right)$ at an excess pressurel,13.

Secondly, to preserve the simplicity of the method of extrapulmonary oxygenation and the use of this method in domestic conditions at the stage of self-and mutual assistance, today there is no alternative to the introduction of a solution of hydrogen peroxide into the stomach ${ }^{13}$.

Third, to improve the effectiveness of protecting the brain from hypoxic damage in conditions of lack of oxygen (hypoxia), simultaneously with the introduction of hydrogen peroxide into the stomach, it is necessary to cool the brain. In other words, there is no alternative for craniocerebral hypothermia.

Fourth, regardless of this, general hypothermia or artificial hibernation can be applied.

The fact is that a decrease in the temperature of human body tissues and warm-blooded animals increases resistance to hypoxia due to a slowdown in the intensity of aerobic metabolism ${ }^{18}$.

But this is not all that can be used to improve the effectiveness of an alternative method of extrapulmonary oxygenation. There is another reserve for protecting brain cells from hypoxic damage. This reserve is hypercentralization of blood circulation. The fact is that nature has endowed people with a natural mechanism of adaptation to hypoxia in the form of centralization of blood circulation. But this adaptive mechanism does not 
completely exhaust all the possibilities available in the body. The fact is that a significant amount of blood is in the extremities, which because of this continue to "take" oxygen from the brain. At the same time, the limbs are several times more resistant to hypoxia than the brain. Therefore, they can be completely deprived of oxygen for 1 to 2 hours, especially if the limbs are cooled 20 .

To illustrate the amount of blood that can circulate in the lower and upper extremities, we can give data that each lower limb can contain a volume of blood that is on average $10 \%$ of the volume of circulating blood in the human body $^{23}$. From this it follows that the 2 lower limbs and 2 upper limbs of a person can contain a total of more than $20 \%$ of the total volume of blood circulating in his body (5 - 7 liters). In other words, a person's feet and hands may contain more than 1 liter of blood. This is true. It is reported that orthostatic deposition of blood in the lower extremities in humans can reach a volume of 1.5 liters of blood 24.

In this regard, it becomes clear that it is possible to increase the survival of brain cells in pulmonary insufficiency and hypoxia by artificially hypercentrating blood circulation. This artificial Hypercentralization of blood circulation can be achieved very simply and reliably in almost any conditions by applying tourniquets to the extremities. However, this raises an artificial ischemia of the extremities. But the limbs can easily withstand this ischemia if they are cooled during this period. To do this, it is enough to lower the temperature in the area of limb ischemia to $+18-+20^{\circ} \mathrm{C}$.

\section{Conclusion}

Progressive hypoxia, which occurs in patients with pneumonia caused by COVID-19, causes the death of brain cells. In conditions of blockage of the respiratory tract with pus and mucus, resuscitation due to pulmonary oxygenation is not very effective. Today, ECMO is used to protect the brain from hypoxic damage. But this is a very dangerous, expensive and complex procedure. In addition, it is not always possible to apply ECMO in a timely manner. Sometimes a few minutes or hours are missing. Moreover, even if ECMO is used, it is always advisable to reduce the duration of this procedure by a few days or hours. In this regard, a modernized method of extrapulmonary oxygenation in combination with general artificial hypothermia and/or local craniocerebral hypothermia is proposed as a reserve and/or alternative to ECMO.

In this regard, as an alternative to ECMO, gastric oxygenation is proposed using a solution of hydrogen peroxide, carbonated with oxygen under excessive pressure. At the same time, it is proposed to perform gastric oxygenation simultaneously with artificial hypercentration of blood circulation, to achieve which it is proposed to use acute limb ischemia, but to create ischemia in the conditions of local hypothermia. Additionally, general artificial hypothermia and/or craniocerebral hypothermia can be used. In this case, the introduction of hydrogen peroxide and oxygen gas into the stomach provides extrapulmonary oxygenation, the creation of acute cold limb ischemia provides artificial hypercentration of blood circulation, and general 
hypothermia and/or craniocerebral hypothermia reduces the brain's need for oxygen. All this together preserves the human brain in conditions of very poor pulmonary oxygenation.

\section{Conflict of interest: None declared.}

\section{Reference}

1. Urakov AL, Urakova NA. COVID-19: What drug can be used to treat a new coronavirus disease and why. J.Bio.Innov. 2020; 9(3): 241-251. https://doi.org/10.46344/JBINO.2020.v09i0 3.02.

2. Velavan TP, Meyer CG. The COVID-19 epidemic. Trop Med Int Health. 2020.

DOI: https://doi.org/10.1111/tmi.13383.

[PubMed].

3. Guan W-j, Ni Z-y, Hu Y, Liang W-h, Ou C-q, He J-x. et al. Clinical Characteristics of Coronavirus Disease 2019 in China. N Engl J Med. 2020 DOl: 10.1056/NEJMoa2002032.

[Google Scholar].

4. Wang D, Hu B, Hu C, Zhu F, Liu $X$, Zhang J. et al. Clinical Characteristics of 138 Hospitalized Patients With 2019 Novel Coronavirus-Infected Pneumonia in Wuhan, China. JAMA. 2020 DOI:10.1001/jama.2020.1585. [PMC free article] [PubMed] [Google Scholar]

\section{Fan E, Sorbo DL, Goligher} EC, et al. An official American Thoracic Society/European Society of Intensive Care Medicine/Society of Critical Care Medicine clinical practice guideline: mechanical ventilation in adult patients with acute respiratory distress syndrome. Am J Respir Crit Care Med. 2017;195(9):1253-1263.
6. Wang JT, Sheng WH, Fang CT, Chen YC, Wang JL, Yu CJ. et al. Clinical manifestations, laboratory findings, and treatment outcomes of SARS patients. Emerg Infect Dis. 2004;10:818-24. [PMC free article] [PubMed] [Google Scholar]

\section{Fielding-Singh V Matthay} MA Calfee CS Beyond low tidal volume ventilation: treatment adjuncts for severe respiratory failure in acute respiratory distress syndrome.Crit Care Med. 2018; 46: 1820-1831.

8. Makhoul M, Bitton-Worms K, Adler Z, Saeed A, Cohen O, Bolotin G. Rambam Maimonides Med J. 2019; 10(2): e0013. Published online 2019 Apr 18. doi: 10.5041/RMMJ.10363.

9. Pappalardo F, Crivellari $\mathbf{M}$. Predicting outcome of venovenous ECMO: look outside the lung! J Thorac Dis. 2018; 10(3): 1356-1360. doi: 10.21037/jtd.2018.02.78.

10. Harvey MJ, Gaies MG, Prosser LA. U.S. and international in-hospital costs of extracorporeal membrane oxygenation: a systematic review. Appl Health Econ Health Policy. 2015;13(4): 341-57. doi: 10.1007/s40258-015-0170-9.

11. Urakov AL. Pus solvents as new drugs with unique physical and chemical properti. Reviews on Clinical Pharmacology and Drug Therapy. 2019; 17 (4): 89 - 95. doi: 10.17816/RCF17489-95.

12. Urakov AL, Urakova NA, Chernova LV. The influence of temperature, atmospheric pressure, antihypoxant and chemical "battery oxygen" on the sustainability of fish in the water without air. International Journal of Applied and Fundamental Research. 2014; 8: 1-5. https://www.elibrary.ru/item.asp? id=21 65 3833. 
13. Urakov AL, Urakova NA. COVID-19: Cause of death and medications. IP International Journal of Comprehensive and Advanced Pharmacology. 2020; 5(2):

14. Urakov AL. Hydrogen peroxide can replace gaseous oxygen to keep fish in hypoxia. International Research Journal. 2017; 5-2 (59): 106 108.

doi:

https://doi.org/10.23670/IRJ.2017.59.109.

15. Yun DJ. Extrapulmonary oxygenation by giving hydrogen peroxide by enema. Yonsei Medical Journal. 1969; 10(2):125-138. Published online February 20, 2002. https://doi.org/10.3349/ymj.1969.10.2.125.

16. Barr J, Livne A, Lushkov G, Vinograd I, Efrati Y, Ballin A, Lahat E, Eshel G. Peritoneal ventilation: an animal model of extrapulmonary ventilation in experimental adult respiratory distress syndrome. Pediatric Research. 1994: 35(6):

682-684. https://www.nature.com/articles/pr19942 88.pdf? origin=ppub.

17. Urakov AL, Urakova NA, Chernova LV. Hydrogen peroxide solution may become a rival gas oxygen during resuscitation. Advances in Current Natural Science. 2014; 12: 198-203. URL: www.rae.ru/use/? section $=$ content $\& O p=s$ how_article\&article_id=10003408.

(available : 01.01.2015).

18. Urakov A, Urakova N. Fetal hypoxia: Temperature value for oxygen exchange, resistance to hypoxic damage, and diagnostics using a thermal imager. Indian J Obstet Gynecol Res 2020;7(2): $232 \quad-238$. https://creativecommons.org/licenses/by $-n c / 4.0 /$.
19. Urakov A, Urakova N, Kasatkin A. Thermal imaging improves the accuracy hemorrhagic shock diagnostics. The concept and practical recommendations. LAP LAMBERT Academic Publishing. 2016:51.

20. Urakov AL, Odiankov EG, Odiankov YuG, Muraviev MF, Kolodkin DE, Volkov VV. Local hypothermia in the treatment of acute arterial obstruction of the limb. Bulletin of surgery.1988; 7: 62 65.

21. Urakov AL. Recipe for temperature. Science and Life. 1989; 9: 38 $-42$.

22. Makdisi G, Wang I-wen. Extra corporeal membrane oxygenation (ECMO) review of a lifesaving technology. J Thorac Dis. 2015; 7(7): E166E176. doi: 10.3978/j.issn.20721439.2015.07.17.

23. Adams J, Albert S. The blood volume in the lower extremities: a technique for its determination utilizing Cr-51 tagged red cells. The Journal of Bone \& Joint Surgery. 1962; 44(3): 489-493.

24. Jabot J, Teboul JL, Richard C, Monnet $\mathbf{X}$. Passive leg raising for predicting fluid responsiveness: importance of the postural change. Intensive Care Med. 2009;35:85-90. doi: 10.1007/s00134-008-1293-3. 